\title{
Evolution of the Pauli spin-paramagnetic effect on the upper critical fields of $\mathbf{K}_{x} \mathrm{Fe}_{2-y} \mathrm{Se}_{2-z} \mathrm{~S}_{z}$ single crystals
}

\author{
F. Wolff-Fabris, ${ }^{1, \S}$ Hechang Lei, ${ }^{2, \dagger, \ddagger}$ J. Wosnitza, ${ }^{1,3}$ and C. Petrovic ${ }^{2, \ddagger}$ \\ ${ }^{1}$ Hochfeld-Magnetlabor Dresden (HLD), Helmholtz-Zentrum Dresden-Rossendorf, D-01314 Dresden, Germany \\ ${ }^{2}$ Condensed Matter Physics and Materials Science Department, \\ Brookhaven National Laboratory, Upton, New York 11973, USA \\ ${ }^{3}$ Institut für Festkörperphysik, TU Dresden, D-01062, Dresden, Germany
}

(Dated: September 2, 2018)

\begin{abstract}
We have studied the temperature dependence of the upper critical fields, $\mu_{0} H_{c 2}$, of $\mathrm{K}_{x} \mathrm{Fe}_{2-y} \mathrm{Se}_{2-z} \mathrm{~S}_{z}$ single crystals up to $60 \mathrm{~T}$. The $\mu_{0} H_{c 2}$ for $H \| a b$ and $H \| c$ decrease with increasing sulfur content. The detailed analysis using Werthamer-Helfand-Hohenberg (WHH) theory including the Pauli spin-paramagnetic effect shows that $\mu_{0} H_{c 2}$ for $H \| a b$ is dominated by the spinparamagnetic effect, which diminishes with higher $\mathrm{S}$ content, whereas $\mu_{0} H_{c 2}$ for $H \| c$ shows a linear temperature dependence with an upturn at high fields. The latter observation can be ascribed to multiband effects that become weaker for higher $\mathrm{S}$ content. This results in an enhanced anisotropy of $\mu_{0} H_{c 2}$ for high $\mathrm{S}$ content due to the different trends of the spin-paramagnetic and multiband effect for $H \| a b$ and $H \| c$, respectively.

PACS numbers: 74.25.Op, 74.25.F-, 74.70.Xa
\end{abstract}

\section{INTRODUCTION}

Since the discovery of $\mathrm{LaFeAsO}_{1-x} \mathrm{~F}_{x}$ with $T_{c}=26$ $\mathrm{K}, \underline{1}$ there has been considerable effort invested in understanding the properties and superconducting mechanism of iron-based superconductors $\stackrel{2-4}{-4}$ Thereby, the temperature dependence of the upper critical field, $\mu_{0} H_{c 2}$, attracts great interests because it provides valuable information on the coherence length, anisotropy, electronic structure, and pair-breaking mechanism. However, iron-based superconductors exhibit a rich diversity in the temperature dependence of $\mu_{0} H_{c 2}$. For FeAs-1111- and FeAs-122-type superconductors, such as $\mathrm{La}(\mathrm{O}, \mathrm{F}) \mathrm{FeAs}$ and $\mathrm{Sr}(\mathrm{Fe}, \mathrm{Co})_{2} \mathrm{As}_{2}$, the upper critical fields can be described using a two-band model $\stackrel{5,6}{6}$ For FeAs111- and FeSe-11-type superconductors, such as LiFeAs and $\mathrm{Fe}(\mathrm{Te}, \mathrm{Se} / \mathrm{S}), \mu_{0} H_{c 2}$ is dominated by Pauli spinparamagnetism.$^{\underline{7}-\underline{9}}$

Studies of the upper critical field in FeSe-122-type superconductors $\left(A_{x} \mathrm{Fe}_{2-y} \mathrm{Se}_{2}\right.$, with $A=\mathrm{K}, \mathrm{Rb}$, Cs, or $\mathrm{Tl}$ ) are rare because of the rather high superconducting transition temperature, $T_{c}$, and concomitantly large zerotemperature critical field. In addition, it is very challenging to handle the air-sensitive samples. Mun et al. studied $\mu_{0} H_{c 2}$ of $\mathrm{K}_{0.8} \mathrm{Fe}_{1.76} \mathrm{Se}_{2}$ up to $60 \mathrm{~T}, \underline{10}$ They found that the upper critical field for $H \| c, \mu_{0} H_{c 2, c}$, increases quasilinearly with decreasing temperature, whereas $\mu_{0} H_{c 2, a b}$ for $H \| a b$ flattens at low temperatures. The anisotropy of upper critical field, $\gamma=H_{c 2, a b} / H_{c 2, c}$, decreases with $T$ and drops to about 2.5 at $18 \mathrm{~K}$. A similar behavior has been observed for $\mathrm{Tl}_{0.58} \mathrm{Rb}_{0.42} \mathrm{Fe}_{1.72} \mathrm{Se}_{2}{ }^{11}$ The analysis of $\mu_{0} H_{c 2}$ using the Werthamer-Helfand-Hohenberg (WHH) formula including Pauli spin-paramagnetism and spin-orbit scattering indicates that spin paramagnetism plays an important role in $\mu_{0} H_{c 2, a b}$, whereas the enhancement of $\mu_{0} H_{c 2, c}$ at low temperatures is likely attributed to multiband effects $\underline{\underline{11}}$
In $\mathrm{K}_{x} \mathrm{Fe}_{2-y} \mathrm{Se}_{2}$, substitution of Se by sulfur suppresses $T_{c} \stackrel{12}{=}$ Preliminary measurements of $\mu_{0} H_{c 2}$ at low fields reveal that $\mu_{0} H_{c 2}$ as well decreases with $\mathrm{S}$ content, thereby showing temperature dependence that can be described well using the simplified WHH model without spin paramagnetism and spin-orbit scattering. $\stackrel{13}{\underline{13}}$ However, the evolution of $\mu_{0} H_{c 2}$ at very high fields and low temperatures is still unclear. In this work, we report on the temperature dependence and anisotropy of upper critical fields for three single crystals with different $\mathrm{S}$ concentration, namely $\mathrm{K}_{0.64} \mathrm{Fe}_{1.44} \mathrm{Se}_{2}(\mathrm{~S}-0), \mathrm{K}_{0.70(7)} \mathrm{Fe}_{1.55(7)} \mathrm{Se}_{1.01(2)} \mathrm{S}_{0.99(2)}$ (S99), and $\mathrm{K}_{0.76(5)} \mathrm{Fe}_{1.61(5)} \mathrm{Se}_{0.96(4)} \mathrm{S}_{1.04(5)}$ (S-104), accessing pulsed magnetic fields up to $60 \mathrm{~T}$. We found that the spin-paramagnetic effect is rather important in $\mu_{0} H_{c 2, a b}$, but, at the same time, multiband effects dominate the temperature dependence of $\mu_{0} H_{c 2, c}$. These effects become weaker with higher S content.

\section{EXPERIMENT}

The single crystals of $\mathrm{K}_{x} \mathrm{Fe}_{2-y} \mathrm{Se}_{2-z} \mathrm{~S}_{z}$ used in this study were grown and characterized as described previously $\underline{12}$ Magnetotrasport experiments in pulsed magnetic fields up to $62 \mathrm{~T}$ were performed at the Dresden High Magnetic Field Laboratory facility, a member of the European Magnetic Field Laboratory. Exposure of the samples to ambient conditions was minimized by handling the samples in a glove box. We have used standard four-contacts technique with $\mathrm{AC}$ currents operating in the $\mathrm{kHz}$ frequency range. The electrical resistance was measured by use of a fast data-acquisition recording system and analyzed with a digital lock-in technique. The contacts were made on freshly cleaved surfaces inside a glove box using silver paint and platinum wires. The contact resistance was between 10 and $50 \mathrm{Ohms}$ and the 

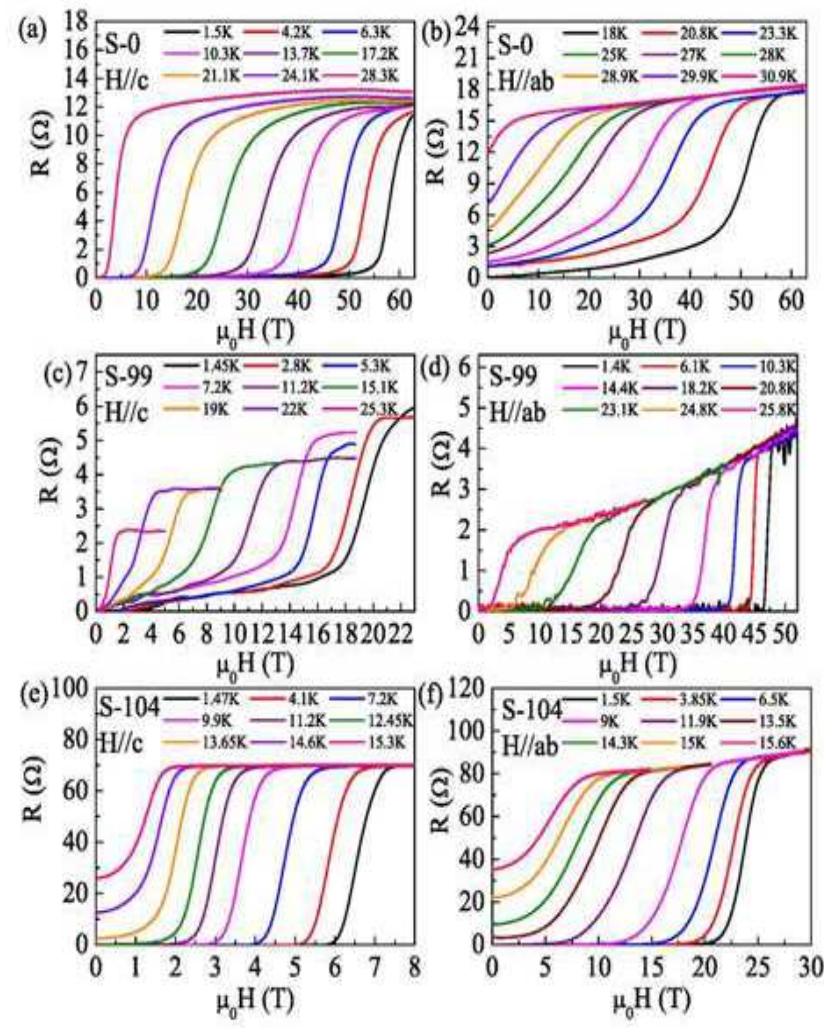

FIG. 1. (Color online) Magnetic-field dependence of the resistance, $R$, of sample S-0 for (a) $H \| c$ and (b) $H \| a b$, of sample S-99 for (c) $H \| c$ and (d) $H \| a b$, and of sample S-104 for (e) $H \| c$ and (f) $H \| a b$ measured at various temperatures.

excitation current was $0.3 \mathrm{~mA}$ which corresponds to the current density of approximately $10^{3} \mathrm{~A} / \mathrm{m}^{2}$. Anisotropic measurements were conducted on the same crystal.

$\mathrm{K}_{x} \mathrm{Fe}_{2-y} \mathrm{Se}_{2}$ samples are intrinsically phase separated into nanoscale magnetic insulating and Josephsoncoupled superconducting regions $\underline{\underline{14}-21}$ The insulating parts of the sample have several orders of magnitude higher resistance $\left(R_{i}\right)$ near $T_{c}$ when compared to the metallic parts $\left(R_{m}\right) \stackrel{22}{2}$ Therefore, near $T_{c}$ and below $R(T) \approx R_{m}(T)$. Recent angular resolved photoemission data showed that the insulating parts of the sample do not contribute to the spectral weight in the energy range

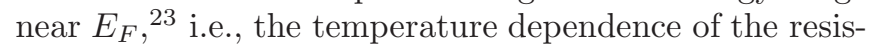
tivity below $T_{c}$ is dominated by the metallic parts of the sample, similar to polycrystals having dense grain boundaries. Since sulfur substitution in $\mathrm{K}_{x} \mathrm{Fe}_{2-y} \mathrm{Se}_{2}$ crystals is uniform,,$\frac{12,24}{2} \mathrm{~S}$ most likely substitutes both superconducting and insulating phase fractions.

\section{RESULTS AND DISCUSSION}

Figure 1 shows the field dependence of the resistance, $R$, of the samples S-0, S-99, and S-104 for $H \| c$ and
$H \| a b$ at various temperatures. Superconductivity is suppressed and the normal state recovered with increasing magnetic fields at constant temperature and the superconducting transitions in $R$ shift to lower magnetic fields at higher temperatures. For some curves we observe a finite resistance in the superconducting state that may be caused either by experimental artifacts or by thermally activated vortex-flux motion. The experimental artifacts may include a degradation of the contacts or sample cracking during the course of the experiment. For all samples, at the same temperature, the transitions for $H \| a b$ occur at much higher fields when compared to those for $H \| c$. This shows that $\mu_{0} H_{c 2, a b}$ is much larger than $\mu_{0} H_{c 2, c}$ and that there exists a large anisotropy in the upper critical fields for all samples. On the other hand, for both field directions, the transitions rapidly shift to lower fields with increasing S content. For example, the superconducting transition at $T \sim 1.5 \mathrm{~K}$ changes from about 60 to $20 \mathrm{~T}$ and finally reaches $7 \mathrm{~T}$ for the samples S-0, S-99, and S-104, respectively. This evidences that $\mathrm{S}$ doping significantly suppresses $\mu_{0} H_{c 2}$, consistent with previous results measured at low fields $\underline{\underline{13}, 25}$

Figure 2 presents the temperature dependence of the resistive upper critical fields, $\mu_{0} H_{c 2}$, of S-0, S-99, and S104 determined from the resistivity drops to $90 \%$ (Onset), $50 \%$ (Middle), and 10\% (Zero) of the normal state resistance, $R_{n}$, for both field directions. The normal-state resistance was determined by linearly extrapolating the field-dependent resistance above the onset of the superconductivity transition. The data taken in low fields are in good agreement for S-104 crystal [Fig. 2(c)]. The S99 crystal had $T_{c} \sim 26 \mathrm{~K}$, somewhat higher than crystal used in low field studies, $\stackrel{13}{=}$ but expected for crystals with that sulfur content $\frac{12}{2}$ For all samples, $\mu_{0} H_{c 2}$ obtained for $H \| a b$ is much larger than for $H \| c$, as mentioned above. The temperature dependence of $\mu_{0} H_{c 2, a b}$ for sample S-0 [Fig. 2(a)] is distinctively different from that of $\mu_{0} H_{c 2, c}$. Close to $T_{c 0}$ (zero-field transition temperature), clearly different slopes are observed in the temperature dependence of $\mu_{0} H_{c 2}$ for both field orientations. With decreasing temperature, the $\mu_{0} H_{c 2, a b}$ curves start to bend downward with a convex shape. In contrast, $\mu_{0} H_{c 2, c}$ exhibits almost linear temperature dependence. These results for the S-0 sample are consistent with previous measurements using a contactless of technique $\stackrel{10,26}{=}$ This suggests the absence of resistive heating in our measurements.

For the sample S-99 [Fig. 2(b)], $\mu_{0} H_{c 2}$ for both field directions show a similar behavior as for S-0 but the absolute values are much smaller than for the pure crystal. Moreover, $T_{c 0}$ also shifts to lower temperature. For sample S-104 [Fig. 2(c)], $\mu_{0} H_{c 2, a b}$ and $\mu_{0} H_{c 2, c}$ exhibit similar saturation trends at low temperatures with different slopes at $T$ close to $T_{c 0}$. When compared to the previous results measured in low fields for sample S-104, $\mu_{0} H_{c 2, c}$ can be well described using the simplified WHH formula, but $\mu_{0} H_{c 2, a b}$ is remarkably smaller than the value predicted $\stackrel{13}{\underline{n}}$ This implies that the temperature de- 

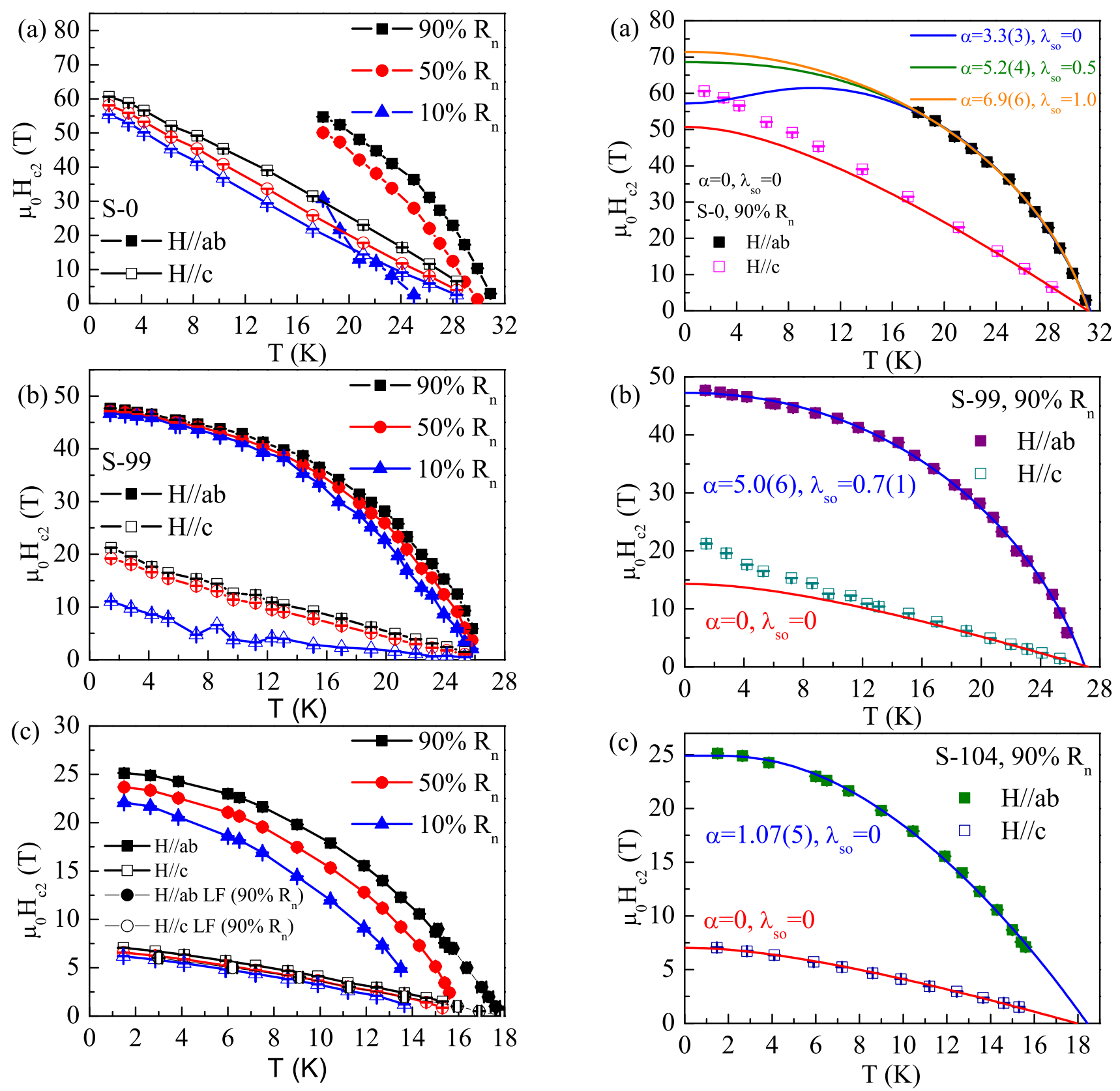

FIG. 2. (Color online) Temperature dependence of the resistive upper critical fields, $\mu_{0} H_{c 2}$ of the samples (a) S-0, (b) S-99, and (c) S-104 for $H \| a b$ (closed symbols) and $H \| c$ (open symbols). Figure 2 includes low field data (LF) taken on S-104 sample used in Ref. 13 for comparison

pendence of $\mu_{0} H_{c 2}$ is influenced by factors outside the simplified WHH model. Similarly, the nearly linear temperature dependence of $\mu_{0} H_{c 2, c}$ for S-0 and S-99 cannot be explained by this model.

As previous studies have shown, spin paramagnetism has a significant influence on the upper critical field of

FIG. 3. (Color online) $\mu_{0} H_{c 2}(T)$ determined from $90 \% R_{n}$ (symbols) and fits using the WHH theory (solid lines) for the samples (a) S-0, (b) S-99, and (b) S-104 at $H \| a b$ and $H \| c$.

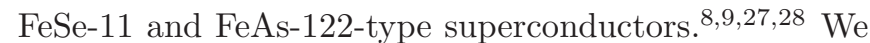
note that the Pauli paramagnetic limiting fields in simplest approximation, $\mu_{0} H_{P}(0)=1.86 T_{c} \stackrel{29,30}{,}$ are about 58.0(2) T, 50.4(2) T, and 33.8(4) T for S-0, S-99, and S-104, respectively. These values are comparable to the zero-temperature $\mu_{0} H_{c 2, a b}$ for these samples. This implies that the spin-paramagnetic effect needs to be considered when analyzing the temperature dependence of 
$\mu_{0} H_{c 2}$, especially for $H \| a b$. Through the Maki parameter, $\alpha$, and $\lambda_{s o} 31$ the effects of Pauli spin paramagnetism and spin-orbit scattering have been included in the WHH theory for a single-band $s$-wave weakly coupled type-II superconductor in the dirty limit $\underline{\underline{32}} \mu_{0} H_{c 2}$ is given by

$$
\begin{gathered}
\ln \frac{1}{t}=\left(\frac{1}{2}+\frac{i \lambda_{s o}}{4 \gamma}\right) \psi\left(\frac{1}{2}+\frac{h+\lambda_{s o} / 2+i \gamma}{2 t}\right) \\
+\left(\frac{1}{2}-\frac{i \lambda_{s o}}{4 \gamma}\right) \psi\left(\frac{1}{2}+\frac{h+\lambda_{s o} / 2-i \gamma}{2 t}\right)-\psi\left(\frac{1}{2}\right),
\end{gathered}
$$

where $\psi(x)$ is the digamma function, $\gamma \equiv\left[(\alpha h)^{2}-\right.$ $\left.\left(\lambda_{\text {so }} / 2\right)^{2}\right]^{1 / 2}$, and

$$
h=\frac{4 \mu_{0} H_{c 2}(T)}{\pi^{2} T_{c}\left(-d \mu_{0} H_{c 2}(T) / d T\right)_{T=T_{c}}} .
$$

When $\alpha>1$, spin paramagnetism becomes essential $\underline{\underline{31}}$ In Fig. 3, $\mu_{0} H_{c 2}$ of S-0, S-99, and S-104, determined using the $90 \% R_{n}$ criterion, together with the fits using formula (11) are shown. The $90 \%$ data were chosen in order to avoid the effects of flux motion and/or sample degradation. When $\lambda_{s o}$ is fixed to $0, \mu_{0} H_{c 2, a b}$ for sample S-0 can be well described with $\alpha=3.3(3)$ indicating strong spin paramagnetism. It should be noted that the fit is not unique and the temperature dependence of $\mu_{0} H_{c 2, a b}$ can as well be described with other fit values of $\alpha$ when $\lambda_{s o}$ is non-zero. This is due to the limited temperature region where data for $\mu_{0} H_{c 2, a b}$ are available. For different combinations of $\alpha$ and $\lambda_{s o}, \mu_{0} H_{c 2, a b}$ varies largely at low temperatures and high fields, below our measurement range. Moreover, in order to describe $\mu_{0} H_{c 2}$ at low fields well, $\alpha$ has to increase when $\lambda_{s o}$ becomes larger because spin-orbit scattering tends to reduce the effect of spin paramagnetism ${ }^{32}$ Accordingly, $\alpha=3.3(3)$ is a lower limit. On the other hand, the WHH model with $\alpha=0$ and $\lambda_{s o}=0$ does not describe the curve well. Thus, even though $\alpha$ and $\lambda_{s o}$ are not uniquely determined by our data, the large $\alpha=3.3(3)$ strongly indicates that spin paramagnetism plays an important role in suppressing superconductivity for $H \| a b$. This sample might be worth to investigate in even higher magnetic fields by using a $100 \mathrm{~T}$ coil in order to explore the evolution of paramagnetic effects for $H \| a b$.

For $H \| c, \mu_{0} H_{c 2, c}$ at low temperatures is enhanced when compared to the WHH model. Such an enhancement cannot be explained by $\alpha>0$ or $\lambda_{s o}>0$. Indeed, it suggests that multiband effect become important when the magnetic field is oriented along the $c$ direction. A similar behavior has been observed in $\mathrm{Tl}_{0.58} \mathrm{Rb}_{0.42} \mathrm{Fe}_{1.72} \mathrm{Se}_{2} \underline{\underline{11}}$

Increasing the $\mathrm{S}$ content to $0.99, \mu_{0} H_{c 2, a b}$ is suppressed to below $50 \mathrm{~T}$ in the whole temperature range [Fig. 3. $\mathrm{b}$ )]. Without considering spin-orbital scattering, the WHH formula cannot describe $\mu_{0} H_{c 2, a b}$ well even when including spin paramagnetism. When the spin-orbit scattering term is included, the fit quality improves significantly and the obtained parameters are $\alpha=5.0(6)$ and $\lambda_{s o}=0.7(1)$.

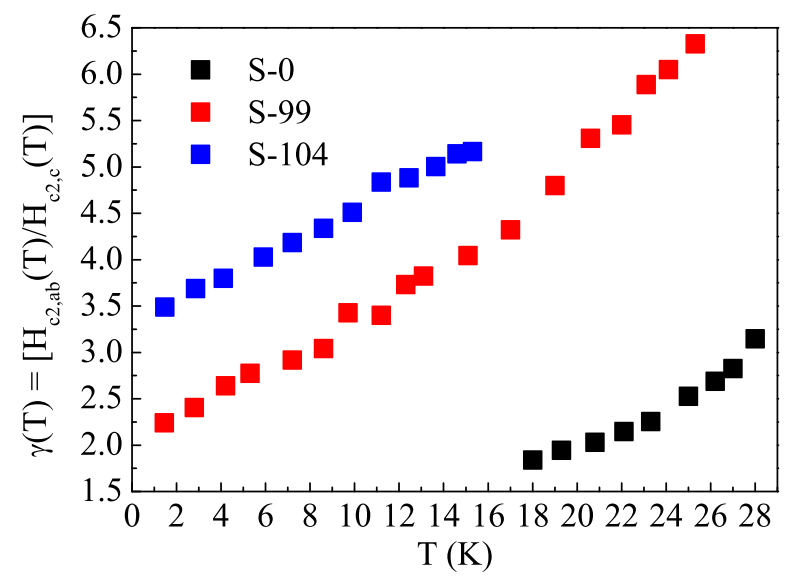

FIG. 4. (Color online) Temperature dependence of the anisotropy, $\gamma=H_{c 2, a b} / H_{c 2, c}$ using the $90 \% R_{n}$ criterion for the samples S-0, S-99, and S-104.

Assuming $\lambda_{s o}=0.7$ for the sample S-0, $\alpha$ in sample S-0 will be larger than in sample S-99. On the other hand, $\mu_{0} H_{c 2, c}$ shows a linear temperature dependence down to about $8 \mathrm{~K}$, below which an upturn appears. As for the pure sample, this upturn cannot be described by use of the simplified WHH model. This shows that multiband effects are still important in sample S-99 for $H \| c$.

For the single-band clean limit, the Maki parameters for $H \| a b$ and $H \| c$ should be related by the ratio of the Fermi velocity $v_{a b}$ in the $a b$ plane to $v_{c}$ along $c$. The cylinder-like Fermi surface in iron-based superconductors results in $v_{a b} \gg v_{c}$. Therefore, this ratio is larger than 1 and the $\alpha$ for $H \| a b$ is expected to be larger than for $H \| c, \stackrel{33}{3}$ i.e., spin paramagnetism is stronger for $H \| a b$ than for $H \| c$. The open electronic orbits along $c$ reduce the orbital-limited upper critical field considerably: 11

With further S substitution [sample S-104, Fig. 31(c)], the WHH model without spin-orbit scattering can describe the temperature dependence of $\mu_{0} H_{c 2, a b}$ well with $\alpha=1.07(5)$. This is somewhat larger than 1, suggesting that spin paramagnetism is still essential. But, the absolute value is much smaller than for the samples S-0 and S-99. Obviously, $\alpha$ decreases with increasing S content, i.e., spin paramagnetism becomes less important. The $\mu_{0} H_{c 2, c}$ can be well described by using the WHH formula without including spin paramagnetism or spinorbit scattering. The slope $-d\left(\mu_{0} H_{c 2, c}\right) / d T \mid$ near $T_{c 0}$ is $0.565(5) \mathrm{T} / \mathrm{K}$, which is very close to the previous results measured at low fields $\stackrel{\underline{13}}{\underline{n}}$ Our results show that the multiband as well as possible spin-paramagnetic effects are largely suppressed in S-104 for $H \| c$.

The anisotropy of the upper critical field, $\gamma=$ $H_{c 2, a b} / H_{c 2, c}$, supplies further information on the effect of $\mathrm{S}$ substitution on the evolution of $\mu_{0} H_{c 2}$. As shown in Fig. 4. $\gamma$ exhibits a similar trend in the temperaturedependence for all three samples: the anisotropy de- 
creases considerably with temperature. For sample S$0, \gamma$ is $\sim 3.1$ at $28 \mathrm{~K}$ and decreases to $\sim 1.8$ at $18 \mathrm{~K}$, a somewhat smaller value than reported in literature.10 For sample S-99, $\gamma$ is about 6.3 at $25 \mathrm{~K}$ and decreases gradually to $\sim 2.2$ at $1.5 \mathrm{~K}$. Finally, for sample S-104, $\gamma$ lies between $\sim 5.2$ at $15 \mathrm{~K}$ and $\sim 3.5$ at $1.5 \mathrm{~K}$. This trend has been observed in all iron-based superconductors ${ }^{33.34}$

The origin of the small anisotropy of the upper critical fields could be caused by a three-dimensional electronic structure, spin paramagnetism, or multiband effects. 34 The notable outcome of our study is that the anisotropy increases with $\mathrm{S}$ content, consistent with previous results measured in the Ginzburg-Landau region where it was speculated that this might be due to a smaller warping of the two-dimensional (2D) Fermi surface (FS) with increasing $\mathrm{S}$ content. 13 The increase of the anisotropy with $\mathrm{S}$ substitution is understandable since orbital pair breaking is more effective near $T_{c 0}, \frac{34}{3}$ and a more $2 \mathrm{D} \mathrm{FS}$ should lead to a larger $\gamma$. However, this work shows that other factors can as well result in an enhanced anisotropy of $\mu_{0} H_{c 2}$ in the high-field low-temperature region. First, spin paramagnetism which usually decreases the anisotropy of the upper critical field at high fields becomes weaker with increasing $\mathrm{S}$ content, thus the suppression of $\mu_{0} H_{c 2, a b}$ is reduced. Second, multiband effects also become weaker at higher $\mathrm{S}$ content, and the slight upturn of $\mu_{0} H_{c 2, c}$ at high fields changes to a saturation behavior described by the WHH model. These two opposite trends contribute to a larger $\gamma$. Since $\alpha$ is proportional to the effective mass in the clean limit, 35 the smaller $\alpha$ with increasing $\mathrm{S}$ content can partially be related to a decrease of the effective mass $\stackrel{33.36}{~ T h e ~ d e c r e a s e ~}$ in $\alpha$ is in agreement with suppression of spin susceptibility and spin excitations in $\mathrm{K}_{x} \mathrm{Fe}_{2-y} \mathrm{Se}_{1-z} \mathrm{~S}_{z} \cdot 37$ In addi- tion, sulfur substitution might also change the electronic structure leading to reduced multiband effects. Further theoretical work is necessary to clarify this.

\section{CONCLUSION}

In summary, we have investigated the upper critical fields of $\mathrm{K}_{x} \mathrm{Fe}_{2-y} \mathrm{Se}_{1-z} \mathrm{~S}_{z}$ single crystals up to $60 \mathrm{~T}$. The $\mu_{0} H_{c 2}$ for both $H \| a b$ and $H \| c$ decreases with increasing $\mathrm{S}$ content. For $H \| a b$, the $\mu_{0} H_{c 2, a b}$ follows the WHH model including strong spin paramagnetism. The $\mu_{0} H_{c 2, c}$ for low $\mathrm{S}$ content shows a behavior that suggests multiband effects and the single-band orbitally limited field gradually becomes dominant at high sulfur content. The anisotropy of $\mu_{0} H_{c 2}$ is enhanced with increasing $\mathrm{S}$ content which can be explained by weakened spin paramagnetism and reduced multiband effects for $H \| a b$ and $H \| c$, respectively.

\section{ACKNOWLEDGEMENTS}

Work at Brookhaven is supported by the Center for Emergent Superconductivity, an Energy Frontier Research Center funded by the U.S. DOE, Office for Basic Energy Science (HL and CP). Part of this work was supported by EuroMagNET II (EU Contract No. 228043). CP acknowledges support by the Alexander von Humboldt Foundation.

$\S$ Current Address: European XFEL GmbH, Notkestrasse 85, 22607, Hamburg, Germany.

$\dagger$ Current Address: Frontier Research Center and Materials and Structures Laboratory, Tokyo Institute of Technology, 4259 Nagatsuta, Midori, Yokohama 2268503, Japan

$\ddagger$ hlei@lucid.msl.titech.ac.jp and petrovic@bnl.gov
1 Y. Kamihara, T. Watanabe, M. Hirano, and H. Hosono, J. Am. Chem. Soc. 130, 3296 (2008).

2 G. R. Stewart, Rev. Mod. Phys. 83, 1589 (2011).

3 E. Dagotto, Rev. Mod. Phys. 85, 849 (2013).

4 Jianping $\mathrm{Hu}$ and Ningning Hao, Phys. Rev. X 2, 021009 (2012).

${ }^{5}$ F. Hunte, J. Jaroszynski, A. Gurevich, D. C. Larbalestier, R. Jin, A. S. Sefat, M. A. McGuire, B. C. Sales, D. K. Christen, and D. Mandrus, Nature 453, 903 (2008).

6 S. A. Baily, Y. Kohama, H. Hiramatsu, B. Maiorov, F. F. Balakirev, M. Hirano, and H. Hosono, Phys. Rev. Lett. 102, 117004 (2009).

7 S. Khim, B. Lee, J. W. Kim, E. S. Choi, G. R. Stewart, and K. H. Kim, Phys. Rev. B 84, 104502 (2011).

${ }^{8}$ H. C. Lei, R. W. Hu, E. S. Choi, J. B. Warren, and C. Petrovic, Phys. Rev. B 81, 094518 (2010).

${ }^{9}$ H. C. Lei, R. W. Hu, E. S. Choi, J. B. Warren, and C. Petrovic, Phys. Rev. B 81, 184522 (2010).

10 E. D. Mun, M. M. Altarawneh, C. H. Mielke, V. S. Zapf, R. Hu, S. L. Bud'ko, and P. C. Canfield, Phys. Rev. B 83, 100514(R) (2011).
11 L. Jiao, Y. Kohama, J. L. Zhang, H. D. Wang, B. Maiorov, F. F. Balakirev, Y. Chen, L. N. Wang, T. Shang, M. H. Fang, and H. Q. Yuan, Phys. Rev. B 85, 064513 (2012).

12 H. C. Lei, M. Abeykoon, E. S. Bozin, K. F. Wang, J. B. Warren, and C. Petrovic, Phys. Rev. Lett. 107, 137002 (2011).

13 H. C. Lei and C. Petrovic, Europhys. Lett. 95, 57006 (2011).

14 D. H. Ryan, W. N. Rowan-Weetaluktuk, J. M. Cadogan, R. Hu, W. E. Straszheim, S. L. Budko, and P. C. Canfield, Phys. Rev. B 83, 104526 (2011).

15 Z. Wang, Y. J. Song, H. L. Shi, Z. W. Wang, Z. Chen, H. F. Tian, G. F. Chen, J. G. Guo, H. X. Yang, and J. Q. Li, Phys. Rev. B 83, 140505 (2011).

16 Y. Liu, Q. Xing, K. W. Dennis, R. W. McCallum, and T. A. Lograsso, Phys. Rev. B 86, 144507 (2012).

17 A. Ricci, N. Poccia, B. Josesph, G. Arrighetti, L. Barba, J. Plaiser, G. Campi, Y. Mizuguchi, H. Takeya, Y. Takano, N. L. Saini, and A. Bianconi, Supercond. Sci. Technol. 24, 082002 (2011).

18 Wei Li, H. Ding, P. Deng, K. Chang, C. Song, Ke He, L. 
Wang, X. Ma, J. P. Hu, X. Chen, and Q.K. Xue, Nature Physics 8, 126 (2012).

19 R. H. Yuan, T. Dong, Y. J. Song, P. Zheng, G. F. Chen, J. P. Hu, J. Q. Li, and N. L. Wang, Sci. Rep. 2, 221 (2012).

${ }^{20}$ D. Louca, K. Park, B. Li, J. Neufeind, and J. Yan, Sci. Rep. 3, 2047 (2013).

21 N. Lazarevic, M. Abeykoon, P. W. Stephens, Hechang Lei, E. S. Bozin, C. Petrovic, and Z. V. Popovic, Phys. Rev. B 86, 054503 (2012).

22 D. P. Shoemaker, D. Y. Chung, H. Claus, M. C. Francisco, S. Avci, A. Llobet, and M. G. Kanatzidis, Phys. Rev. B 86, 184511 (2012).

${ }^{23}$ M. Yi, D. H. Lu, R. Yu, S. C. Riggs, J.-H. Chu, B. Lv, Z. K. Liu, M. Lu, Y.-T. Cui, M. Hashimoto, S.-K. Mo, Z. Hussain, C. W. Chu, I. R. Fisher, Q. Si, and Z.-X. Shen, Phys. Rev. Lett. 110, 067003 (2013).

24 Hechang Lei and C. Petrovic, Phys. Rev. B 84, 052507 (2011).

25 J. J. Ying, X. F. Wang, X. G. Luo, A. F. Wang, M. Zhang, Y. J. Yan, Z. J. Xiang, R. H. Liu, P. Cheng, G. J. Ye, and X. H. Chen, Phys. Rev. B 83, 212502 (2011).

${ }^{26}$ V. A. Gasparov, A. Audouard, L. Drigo, A. I. Rodigin, C. T. Lin, W. P. Liu, M. Zhang, A. F. Wang, X. H. Chen, H.
S. Jeevan, J. Maiwald and P. Gegenwart, Phys. Rev. B 87, 094508 (2013).

27 V. A. Gasparov, F. Wolff-Fabris, D. L. Sun, C. T. Lin and J. Wosnitza, JETP Lett. 93, 26 (2011).

28 V. A. Gasparov, L. Drigo, A. Audouard, D. L. Sun, C. T. Lin, S. L. Bud'ko, P. C. Canfield, F. Wolff-Fabris and J. Wosnitza, JETP Lett. 93, 667 (2001).

29 B. S. Chandrasekhar, Appl. Phys. Lett. 1, 7 (1962).

30 A. M. Clogston, Phys. Rev. Lett. 9, 266 (1962).

31 K. Maki, Phys. Rev. 148, 362 (1966).

32 N. R. Werthamer, E. Helfand, and P. C. Hohenberg, Phys. Rev. 147, 295 (1966).

33 H. C. Lei, K. F. Wang, R. Hu, H. J. Ryu, M. Abeykoon, E. S. Bozin, and C. Petrovic, Sci. Technol. Adv. Mater. 13, 054305 (2012).

34 J.-L. Zhang, L. Jiao, Y. Chen, and H.-Q. Yuan, Front. Phys. 6, 463 (2011).

35 A. Gurevich, Rep. Prog. Phys. 74, 124501 (2011).

${ }^{36}$ K. F. Wang, H. C. Lei, and C. Petrovic, Phys. Rev. B 84, 054526 (2011).

37 D. A. Torchetti, T. Imai, H. C. Lei and C. Petrovic, Phys. Rev. B 85, 144516 (2012). 